\title{
IFR
}

Actes de colloques

$\mathbf{N}^{\circ} 32$

\section{L'ENTREPRISE ET L'ART}

Sous la direction de

Alexandra MENDOZA-CAMINADE

Colloque du CDA du 18 novembre 2016

PRESSES DE L'UNIVERSITÉ TOULOUSE 1 CAPITOLE 


\section{L'ENTREPRISE AMATRICE D'ART, L'ENTREPRISE MÉCÈNE :}

\section{Le denier de l'art}

Hélène POUJADE

Maître de conférences à l'Université Toulouse Capitole Centre de Droit des Affaires (CDA)

«(M)algré son nom glorieux, la puissance qu'on lui prête et son geste théâtral, la création ne peut pas survivre par soi-même. Elle meurt sans mécène et ne vit que de lui $»^{1}$.

La défiance. Dès lors, à l'image des fidèles qui, par leur contribution au « denier du culte », permettent à l'Église d'assumer sa mission, qu'en est-il de la contribution des entreprises au «denier de l'art» pour asseoir sa fonction de création et de diffusion de la vie de l'esprit $^{2}$ ?

Mais avant d'explorer «les voies possibles du financement» par l'entreprise amatrice d'art, encore faut-il, en préalable, s'assurer que cette idée même d'une «entreprise mécène », telle une généreuse fidèle, puisse être empruntée!

Or, cela n'a rien d'évident tant l'expression retenue présente son lot de contradictions. N'enseigne-t-on pas que l'entreprise, qu'elle soit sous forme

\footnotetext{
${ }^{1}$ M. Serres, Le couple générique de l'histoire, Le mécénat dans l'histoire, Actes du colloque du 21 mars 1989, Fondation EDF, cité dans J.-P. Alline et R. Carrier (dir.), La culture au risque du marché, le mécénat face à ses acteurs, L'Harmattan, 2010.

${ }^{2}$ Image empruntée à J. Rigaud, Les deniers du rêve : essai sur l'avenir des politiques culturelles, Grasset, 2001.
} 
individuelle ou sociétaire, est transcendée par l'idée de lucre? En ce sens, l'absence de contrepartie, consubstantielle au mécénat, contrarierait toute perspective de financement de l'art par l'entreprise. Ce but non lucratif qualifiant d'autres groupements ${ }^{3}$, il y aurait donc fort à penser que cette vocation philanthropique reste de leur domaine réservé ; à l'exclusion des entreprises.

Défendre l'idée d'une «entreprise philanthropique» paraît en ce sens compromis tant la charité sied mal avec sa fonction naturelle.

Pourtant, cette vision manichéenne selon laquelle, d'un côté, les pouvoirs publics serviraient l'intérêt général, tandis que, de l'autre, l'entreprise serait animée par la seule quête du profit, doit être abandonnée. C'est d'ailleurs en cherchant à comprendre les motivations des entreprises mécènes que la pureté de la doctrine philanthropique s'effrite. Dépassant la mission économique des entreprises, il est désormais acquis que cet acte de générosité, rarement intuitif, est souvent conçu comme une véritable stratégie de communication. Par lui, l'entreprise cherche non seulement à optimiser son impact sur la cause soutenue, mais encore à créer une valeur immatérielle. L'animation culturelle devient alors le point d'orgue de son image 4 . Au-delà, ne négligeons pas l'enjeu résidant dans les différentes incitations fiscales vouées à encourager l'intervention des entreprises au chevet de l'art. Elles sont d'ailleurs devenues l'un des éléments caractéristiques de son régime ${ }^{5}$, y compris à l'échelon européen ${ }^{6}$. Ces « niches »

\footnotetext{
${ }^{3}$ En l'occurrence, les associations de la loi du $1^{\mathrm{er}}$ juillet 1901 relative au contrat d'association (art. 1).

${ }^{4}$ En ce sens, J.-M. Décaudin, «Marketing artistique et culturel des entreprises », voir supra dans cet ouvrage.

${ }^{5}$ En ce sens, A. De Bissy, «Optimisation fiscale des sociétés et marchés de l'art », voir infra dans cet ouvrage. Comp. : CGI, art. 200 (particuliers), CGI, art. 238 bis (entreprises) et CGI, art. 885-0 V bis (ISF) modifié par la loi nº 2007-1223 du 21 août 2007 en faveur du travail, de l'emploi et du pouvoir d'achat (dite « loi TEPA ») pour permettre aux redevables de l'ISF de déduire dans la limite annuelle de $50000 €, 75 \%$ des dons notamment effectués au profit notamment des fondations reconnues d'utilité publique et des établissements d'enseignement artistique publics ou privés (C. de l'éducation, art. L. 216-2, L. 216-3, L. 312-6 à L. 312-8 et L. 361-5, BOI 7 S-5-08 n 61 du 9 juin 2008), d'intérêt général, à but non lucratif (Voir à ce sujet : Article 885-0 V bis $\mathrm{A}$ du CGI et B.O.I. 7 S-5-08, $\mathrm{n}^{\circ} 61$ du 9 juin 2008).

${ }^{6}$ Sans nul doute la philanthropie transnationale bénéficie non seulement l'égalité de traitement reconnue en matière de dons transnationaux (CJCE, 27 janvier 2009, grande chambre, aff. C-318/07) - ainsi, les donateurs à l'origine de dons en faveurs d'organismes éligibles mais situés hors de leur État de résidence peuvent se prévaloir de l'application du traitement fiscal de faveur: exonérations ou réductions d'impôts (consacré par la loi de finance rectificative pour 2009 (article 35) modifiant les articles 200, 238 bis et 885-0 V bis-A du CGI)-, mais encore de l'effectivité du dispositif Transnational
} 
font florès. Car, si l'intérêt commercial ou fiscal que poursuit l'entreprise donatrice ne constitue pas le motif décisif de son engagement, cela ne signifie pas pour autant qu'ils doivent être tus. Loin de se réduire à un amour éthéré des formes esthétiques, l'action du mécène repose sur ces diverses considérations.

Le don serait ainsi paré de nombreuses vertus ! Tandis que l'entreprise peut être instrumentalisée par l'art, l'inverse est également vrai. Levons donc le voile sur cette antinomie de "l'entreprise mécène» qui n'est, somme toute, qu'apparente.

La méfiance. L'intrusion de l'élan privé dans la chose artistique procède cependant d'une lente évolution. Dans notre pays, il était en effet courant de penser qu'elle ressortait du domaine réservé de l'État. Puis, au moment du reflux des financements publics, la diversification des ressources est devenue un enjeu crucial pour les milieux artistique et culturel. La problématique du « denier de la culture » s'est ainsi posée avec une particulière acuité alors qu'il pouvait être craint que, faute de moyens, cette «exception française», illustration du rayonnement intellectuel de la France dans le monde, finisse par céder.

L'État-providence, mécène initiateur et régulateur du monde des Arts, a alors eu beau jeu de céder sa place à de nouveaux acteurs. Outre ces impératifs budgétaires, il ne faut pas non plus négliger les vives critiques adressées à l' « étatisation des arts et de la culture ». Ces deux éléments ont indubitablement conduit à son éclipse.

Retrait de l'État, certes; il n'est pourtant point de désengagement! Son affaiblissement ne conduit pas à son effacement. L'État est alors devenu médiateur, sinon moteur de la participation des initiatives privées à la définition de l'intérêt général en multipliant les dispositifs incitatifs et protecteurs ${ }^{7}$ comme

Giving Europe (TGE), même si, en France, la réduction fiscale consentie dans ce cadre ne concerne que l'impôt sur le revenu.

${ }^{7}$ Voir not. : Loi n ${ }^{\circ}$ 2016-925 du 7 juillet 2016, relative à la liberté de la création, à l'architecture et au patrimoine (art. 3 : «La politique en faveur de la création artistique poursuit les objectifs suivants : (...) « $15^{\circ}$ Encourager les actions de mécénat des particuliers et des entreprises en faveur de la création artistique et favoriser le développement des actions des fondations reconnues d'utilité publique qui accompagnent la création). 
en diversifiant les structures juridiques des établissements publics ${ }^{8}$. Or, derrière chacune de ces initiatives, il s'agit encore d'associer l'Etat au soutien de l'Art. Subvention, incitations, décentralisation, sont autant d'outils de relance du mécénat dans lesquels s'inscrit l'appareil d'État.

Autre chose est en revanche de faire appel à l'investissement privé.

Ce changement de paradigme résulte d'une maturation relativement longue. Forte de sa tradition étatique, la France s'était longtemps contentée de tolérer un mécénat spontané, avant que le ministre chargé des affaires culturelles, André Malraux, affirmât en 1965 vouloir " provoquer en France un véritable mécénat culturel à l'instar de ce qui existe à l'étranger, notamment aux États-Unis ${ }^{9}$. La prétention monopolistique de l'État dans l'Art devait donc finir par céder. Germée dans l'antichambre de ce cabinet ministériel, enrichie sous l'impulsion de l'ADMICAL ${ }^{10}$, cette idée va éclore dans la loi $n^{\circ}$ 87-571 du 23 juillet 1987 sur le développement du mécénat (dite Loi Léotard).

L'onction. Ce texte, largement inspiré d'un rapport sur le mécénat en France, soulignant qu'il fallait " engager l'entreprise plutôt que désengager l'Etat ${ }^{11}$, officialise la logique de cofinancement des politiques culturelle, fruit d'une collaboration entre le privé, à l'endroit des entreprises et des particuliers, et le public, en l'occurrence l'État, y compris à travers ses multiples déclinaisons. Car, il ne faut pas négliger qu'en mettant en place diverses incitations fiscales consistant principalement à renoncer à la perception de ressources, l'État demeure un acteur incontournable du financement de l'art.

Pour sortir de la crise, il s'agissait donc de remettre en cause les rapports public / privé en procédant à l'union de deux réalités prétendument opposées : la culture et les affaires. Toutefois, certainement par crainte d'une privatisation

\footnotetext{
${ }^{8}$ Tels les Groupements d'Intérêt Public (GIP) culturels, le syndicat mixte, les Sociétés d'Economie Mixte, les régies et les diverses formes d'établissement public dont l'Établissement Public de Coopération Culturelle (EPCC) constitué par une collectivité territoriale ou un établissement public de coopération intercommunale (EPCI) pour gérer un service public culturel (CGCT, art. L. 1431 et s.).

${ }^{9}$ C. Bryon, « Petite histoire des mesures prises en faveur du mécénat sous la Vème République (19592002) », in Amitiés internationales André Malraux, https://www.andremalraux.com.

${ }^{10}$ Association pour le Développement du Mécénat Industriel et CommerciAL, créée en 1979.

11 A.-D. Perrin, Mécénat français, Rapport pour le Ministère de la Culture et de la Communication, Paris, 1990.
} 
de l'art, de sa "mercantilisation», ce premier pas en faveur d'un mécénat entrepreneurial présenta un cadre juridique et fiscal étroit et lacunaire. Bien que paré de nombreuses vertus, le mécénat n'y est pas reconnu en tant que notion.

La définition du mécénat sera d'abord fiscale, alors identifié comme «le soutien matériel apporté, sans contrepartie directe de la part du bénéficiaire, à une œuvre ou à une personne pour l'exercice d'activités présentant un intérêt général », auquel la culture peut incontestablement être rattachée ${ }^{12}$. Don, absence de contrepartie, intérêt général, voici les trois critères de sa définition au sein desquels, le deuxième mérite l'attention. Dans la nébuleuse de termes : mécène, partenaire, parrain, lui seul permet de départir le mécénat, en tant que véritable don, placé sous le signe de la discrétion, $d u$ parrainage ou «sponsoring», considéré comme une prestation, "un soutien matériel apporté à une manifestation, à un produit ou une organisation, en vue d'en retirer un bénéfice direct» ${ }^{13}$ puisqu'inscrit dans une démarche commerciale aux retombées quantifiables et économiquement vérifiables.

Sans nul doute, le mécénat se prête davantage à la culture que le parrainage, même si elle s'en accommode souvent. Ainsi, la publication du Baromètre 2016 $d u$ mécénat révèle qu'au sein des domaines prioritairement soutenus par les entrepreneurs mécènes, la culture représente $24 \%$, captant $15 \%$ du budget global du mécénat, soit environ 500 millions d'euros ${ }^{14}$. C'est dire si l'intérêt des entreprises pour la culture ne se dément pas! L'avantage est d'ailleurs conforté par les infléchissements subis par la conception de l'intention libérale, celle en principe attendue du mécène, pour la rendre plus accueillante ${ }^{15}$.

Pourtant, malgré cette faveur, il ne faut pas s'y méprendre ! Prétendre au mécénat culturel en cas de parrainage peut s'avérer dangereux ${ }^{16}$.

\footnotetext{
${ }^{12}$ Arrêté du 6 janvier 1989 relatif à la terminologie économique et financière. Comp. : P. Fronton, «Culture et mécénat: attention danger ! ", Juris art 2016, n 34, p. 3.

${ }^{13}$ Selon l'article 39-I-7e du Code général des impôts, le terme de parrainage doit être réservé aux « dépenses engagées dans le cadre de manifestations de caractère [...] culturel ou concourant à la mise en valeur du patrimoine artistique [...] ou à la diffusion de la culture [et] de la langue françaises, lorsqu'elles sont exposées dans l'intérêt direct de l'exploitation ».

${ }^{14}$ Admical, Le mécénat d'entreprise en France, mai 2016.

${ }^{15}$ Ainsi le droit fiscal fait preuve d'une certaine tolérance en admettant certaines contreparties (dont, par ex. l'indication du nom du donateur) sans que cela ne remette en cause la qualification de mécénat (ex. en matière sportive, voir : Instruction fiscale, 4 C2.00).

${ }^{16}$ Pour éviter telle dérive, ce glissement terminologique, la pratique du rescrit fiscal peut s'avérer utile.
} 
Les déclinaisons. Ainsi défini, il convient d'en observer les multiples déclinaisons.

Ce mécénat culturel connaît en premier lieu de plusieurs formes : alors que le mécénat financier est le plus visible (subventions, legs, numéraire...) et le plus dynamique (à l'image du « mécénat participatif » ${ }^{17}$, du don par SMS ${ }^{18}$ ) qu'il s'agisse de financer une exposition, un atelier, l'acquisition d'une œuvre d'art ou de restaurer des objets d'art, le mécénat en nature (apports des tableaux de collectionneurs (peintures, dessins et sculptures), de biens, de marchandises, mise à disposition de locaux, de matériels, ...), comme le mécénat de compétences (mise à disposition gratuite de personnels sur un chantier de rénovation par exemple ${ }^{19}$, prestations, savoir-faire), ne doivent pas être négligés. Car, si le mécénat financier est ouvert à tous, particuliers comme entreprises, ces dernières semblent en revanche plus aptes à intervenir sous ses deux autres formes.

Mais le mécénat culturel procède en second lieu de différents modes de gestion selon qu'il s'opère en "régie directe » ou nécessite l'intermédiation d'une structure tierce, tels les clubs, associations, «sociétés d'amis $»^{20}$, fondations, fonds de dotation... étant observé que ces deux modalités peuvent se combiner utilement ${ }^{21}$, et ce, au plan national comme local, à travers notamment un «mécénat de proximité ».

C'est dire si la palette des interventions au soutien de l'Art est riche en gammes, variée de tons et diverse en intensités !

\footnotetext{
${ }^{17}$ CMF, art. L. 511-6, $7^{\circ}$ créé par Ordonnance ${ }^{\circ} 2014-559$ du 30 mai 2014, complété par le décret $n^{\circ}$ 2014-1053 du 16 septembre 2014 - Comp. : Décret n ${ }^{\circ}$ 2016-1453 du 28 octobre 2016 relatif aux titres et aux prêts proposés dans le cadre du financement participatif (JORF ${ }^{\circ} 0254$ du 30 octobre 2016).

${ }^{18}$ CMF, art. L. 521-3, I, $2^{\circ}$, créé par la loi n ${ }^{\circ} 2016-1321$ du 7 octobre 2016 (art. 94).

${ }^{19}$ Telle que la restauration par l'entreprise de construction Eiffage des colonnes de Buren au PalaisRoyal à Paris.

${ }^{20}$ Telles que la Société des amis du Louvre ou l'Association pour le rayonnement de l'Opéra national de Paris.

${ }^{21}$ Ainsi, si les artistes, personnes physiques, ne peuvent pas bénéficier du mécénat en régie directe des entreprises, ni du mécénat des particuliers, ils peuvent recevoir des subventions de la part de fondations d'entreprises ou de fondations reconnues d'utilité publique.
} 
Mais lorsque sonne l'heure du choix, force est d'observer que les entreprises désireuses de soutenir un projet culturel se satisfont le plus souvent d'un cadre traditionnel, rationnel, sécurisant, pour se couler dans le moule des fonds et fondations de telle sorte que ces instruments constituent l'une des voies privilégiées du mécénat d'entreprises. En témoigne notamment l'accélération de leur croissance, spécialement ces cinq dernières années. Au sein des huit formes coexistant à ce jour en France, quatre d'entre elles sont en effet susceptibles d'être empruntées pour accomplir ce mécénat culturel ${ }^{22}$. Entre la structure la plus institutionnelle, la fondation reconnue d'utilité publique $e^{23}$, et la plus souple : le fonds de dotation ${ }^{24}$, s'immiscent la fondation sous égide ou abritée $e^{25}$ et la fondation d'entreprise ${ }^{26}$.

Plus qu'un inventaire, chacune d'elles traduit les rapports qui se nouent entre l'entreprise et l'art. Plutôt que s'échiner à confronter le monde de la philanthropie à celui des entreprises pour savoir qui est l'humble servant de l'autre, observons comment le législateur est parvenu à les rapprocher. Tandis que, dans un premier temps, il s'est agit pour le mécénat culturel d'entreprise de s'acclimater des véhicules traditionnels, les plus récents participent quant à eux d'un renouvellement de conceptions plus conforme à la logique d'entreprise.

Car, si l'argent est un moyen pour la culture (I), force est de constater que la culture est devenue un moyen pour l'argent (II).

\footnotetext{
${ }^{22}$ Tableau comparatif des huit formes de Fondations, Aklea société d'avocats, JCPE 2014, n 8, 1095.

${ }^{23}$ Loi n ${ }^{\circ}$ 87-571, 23 juill 1987, sur le développement du mécénat. Voir not. : A. Gobin et J.-L. Monnot, «Fondations : la nouvelle donne (Aspects juridiques et fiscaux de la loi du 23 juillet 1987) », JCP N 1987, I, 344 ; M. Pomey, Traité des fondations d'utilité publique, préc. ; J. Imbert, Aperçu historique sur les fondations en droit français, Le droit des fondations en France et à l'étranger : Études Doc. fr., $\mathrm{n}^{\circ}$ 4879, 1989, p. 21.

${ }^{24}$ Loi de modernisation de l'économie $\mathrm{n}^{\circ} 2008-776$ du 4 août 2008 créant les fonds de dotation (article 140). On en dénombre 2226 au 31 déc. 2016 (Observatoire de la Fondation de France).

${ }^{25}$ Loi $\mathrm{n}^{\circ}$ 90-559 du 4 juillet 1990 modifiant les dispositions de la loi du 23 juillet 1987 sur le développement du mécénat et institutionnalisant les fondations abritées. On en dénombre 1229, hors fondations abritées à l'Institut de France, au 31 déc. 2016 (Observatoire de la Fondation de France).

${ }^{26}$ Ibid. On en dénombre 374 au 31 déc. 2016 (Observatoire de la Fondation de France).
} 


\section{L'entreprise au service de l'art}

Les véhicules historiques du mécénat d'entreprise. Tandis que la fondation reconnue d'utilité publique constitue le modèle de référence (A), force est d'admettre qu'elle n'est pas adaptée à toutes les situations et exige certaines prédispositions. Les contraintes inhérentes à sa création et à son fonctionnement ont ainsi conduit à opter pour un modèle subalterne : la fondation sous égide (B).

\section{A. Le véhicule institutionnel du mécénat d'entreprise}

L'originelle : La fondation RUP. Silencieuse sur la notion de mécène, la loi de 1987 susvisée avait en revanche définit avec soin son principal instrument, en l'occurrence la fondation reconnue d'utilité publique (RUP) ${ }^{27}$. À la suivre, elle serait ainsi "l'acte par lequel une ou plusieurs personnes physiques ou morales $^{28}$ décident l'affectation irrévocable de biens, droits ou ressources à la réalisation d'une œuvre d'intérêt général et à but non lucratif» (art. 18). Contrairement au groupement de personnes que sont les associations ou les sociétés, il s'agit d'un groupement de biens affectés à une œuvre d'intérêt général à but non lucratif. Ainsi, la fondation RUP passe par un premier geste d'abandon, celui du dessaisissement total d'un patrimoine ${ }^{29}$, dès lors que «l'affectation est irrévocable».

Si du point de vue de son fondateur, " la fondation est une solution économique, politique, philosophique, mais pas fiscale $»^{30}$, elle l'est pour les donateurs. Outre la capacité de collecte très étendue qu'offre ce statut, en

\footnotetext{
${ }^{27}$ Tel est le statut emprunté par la Fondation Bettencourt Schueller, créée en 1987 (ayant not. soutenu le film du réalisateur Jacques Perrin, Le Peuple migrateur).

${ }^{28}$ Ce texte s'adresse indistinctement aux personnes morales de droit privé (ex : associations, entreprises, mutuelles, congrégations, etc.) et de droit public (collectivités territoriales, établissements publics, etc.).

${ }^{29}$ Ce texte intéresse tant les « biens » (qu'ils soient meubles : numéraire, parts sociales, collections... ou immeubles : immeubles de rapport, château, toute propriété foncière, domaine agricole ou forestier...) que les « droits » (sur une œuvre, un brevet...) ainsi que toute « ressources ».

${ }^{30}$ Mots empruntés à Antoine de Galbert, fondateur de la Maison Rouge, qui explique fort justement que s'il ne paie pas d'impôts sur cet argent, " c'est pour la simple raison qu'il ne lui appartient plus » !
} 
permettant notamment de recevoir des subventions, des dons, des legs, il ouvre en effet aux généreux donateurs le bénéfice d'avantages fiscaux ${ }^{31}$.

Malgré un modèle de fonctionnement relativement simple - puisque les revenus générés par l'immobilisation de l'importante dotation initiale du capital de la fondation ${ }^{32}$ doivent lui permettre d'accomplir la mission qu'elle s'est donnée -, et une capacité étendue, l'élan initial s'est pourtant heurté aux complexités de leur création. Au-delà de la rigidité de leur statut, ainsi conçu pour drainer les fonds privés, l'esprit très institutionnel qui les inspire obéit à une surveillance administrative permanente. Car, si la fondation relève, en tant que personne morale, du droit privé, elle n'en demeure pas moins assujettie, du point de vue de sa création, aux contraintes de la tutelle administrative, sous le contrôle du Conseil d'État ${ }^{33}$.

Certes, «puisqu'il faut protéger non seulement le public, mais aussi le Trésor public, il est normal que des précautions soient prises $»^{34}$. Cependant, la rigueur juridique et financière de cette procédure, véritable chemin de croix à parcourir par tout candidat à la création d'une fondation d'utilité publique, risquait d'étouffer les initiatives qui avaient suscité le renouveau du mécénat en France. Différentes réformes sont alors intervenues pour alléger les modalités de sa constitution ${ }^{35}$.

${ }^{31}$ Ce premier dispositif permet en effet aux entreprises de déduire leurs dons de l'impôt sur les bénéfices, à tout le moins dans la limite de $0,325 \%$ de leur chiffre d'affaires.

${ }^{32}$ La dotation initiale doit être au minimum de $762200 €$. Elle procède de deux modalités :

- soit du vivant de la personne physique ou morale, sous la forme d'une donation entre vifs devant notaire ou d'une donation temporaire d'usufruit.

- soit après le décès de la personne physique, par voie testamentaire : Contrairement à tous les usages, il est ainsi possible d'établir un testament en faveur d'une fondation, personne morale, qui n'existe pas encore et sous la condition qu'elle obtienne la reconnaissance d'utilité publique. Le dépôt de cette demande doit être accompli par l'exécuteur testamentaire dans le délai d'un an à compter de la date d'ouverture de la succession.

${ }^{33}$ Sa mission d'intérêt général lui est reconnue par décret en Conseil d'État après que ses comptes aient été vérifiés par l'organisme de tutelle. L'examen de sa solidité financière repose sur la présentation d'un budget prévisionnel pour les 3 premiers exercices comptables, afin de déterminer si sa dotation « est suffisante pour (qu'elle) remplisse la mission qu'elle s'est donnée ».

${ }^{34}$ Rapp. J. Lang, 26 avr. 1990 : JO Sénat CR, 27 avr. 1990, p. 584.

${ }^{35}$ D'une part, la loi du $1^{\text {er }}$ août 2003 a assoupli les conditions de libération du capital en prévoyant la possibilité d'un engagement sur un financement pluriannuel, consistant à verser la dotation initiale en plusieurs fractions sur une période maximum de 10 ans (auparavant 5 ans). Ce délai de 10 ans court à compter de la date de publication au Journal officiel du décret qui lui accorde la reconnaissance d'utilité 
Pour autant, le manteau prestigieux de la fondation d'utilité publique reste adapté pour des projets significatifs.

\section{B. Le véhicule auxiliaire du mécénat d'entreprise}

La déclinaison: Les fondations sous égide. Solution peut alors être recherchée dans un autre type de structure : les "fondations abritées", autrement dénommées "fondations sous égide». Ces dernières, privées de capacité juridique, sont accueillies au sein de fondations d'utilité publique et gérées par elles. Elles empruntent ainsi le manteau des fondations d'utilité publique sans avoir à souffrir de leurs lourdeurs.

Outre la souplesse de leur création et de leur gestion, l'enjeu de ces fondations abritées consiste à bénéficier, par capillarité, de la capacité juridique et, partant, des possibilités et avantages offerts par la fondation «abritante » RUP.

Près de 50 fondations sont à ce jour abritantes. Parmi elles, l'Institut de France ainsi que la Fondation de France jouent un rôle tout à fait singulier dans le mécénat d'art. La première, créée en 1795, abrite près d'un millier de fondations sous égide, dont notamment l'Académie des beaux-arts. La seconde en comptabilise plus de 750, dont l'une, devenue mythique, La fondation Cartier qui, crée en 1984 par Alain-Dominique Perrin, vise à favoriser le développement de la création contemporaine et à en diffuser la connaissance.

Malgré ce succès, l'absence de personnalité juridique de la fondation abritée peut cependant devenir un poids en raison de leur manque d'autonomie. Sous l'angle décisionnel, d'abord, dès lors que le périmètre de leur mission ne saurait être plus large que celui de la fondation abritante. Sous l'aspect administratif et financier, ensuite, dès lors que sa gestion relève, par convention,

publique (cf. art. 4, modifiant l'art. 18-1 de la loi du 23 juillet 1987). D'autre part, alors qu'il était consulté sur l'approbation des statuts types de la FRUP (L'adoption de ces statuts types, en théorie « recommandés » par le Conseil d'État sont, en pratique, imposés aux fondateurs qui doivent les adopter au mot près, sauf à justifier de tout écart (G. Russo, « Pérenniser les fonds de dotation et fondations ", Juris art 2015, $\mathrm{n}^{\circ} 29$, p.30 et s.), le Conseil d'État a instauré dans un avis du 13 mars 2012 une présomption de garantie de solidité financière «lorsque la dotation atteint au moins un montant d'un million et demi d'euros $»$. 
de la responsabilité de l'institution qui les abrite et en contrepartie de laquelle cette dernière perçoit une rémunération. Selon une approche patrimoniale, enfin, puisque les biens, droits et ressources affectés à la fondation abritée ne sont pas transférés juridiquement dans un patrimoine distinct de celui de la fondation abritante, de telle sorte qu'ils constituent, pour cette dernière, des biens propres, quoique souvent assortis d'une charges ${ }^{36}$.

Utile, le manteau juridique de la fondation d'utilité publique peut se révéler trop étroit, sinon trop lourd, à l'usage. Quant aux fondations sous égide, même si elles ne déméritent pas, la réponse qu'elles apportent reste cependant insatisfaisante. D'autres voies méritent d'être explorées.

\section{L'art au service de l'entreprise}

De la norme au philanthropisme. À la faveur d'un élargissement de la gamme des instruments juridiques offerts aux entreprises pour accomplir leur mécénat, sont nées des structures à caractère privé. Accompagnant le renouvellement de conception de la vocation des chefs d'entreprises, elles visent à leur donner les moyens d'assumer la responsabilité de définir l'intérêt général. Lorsqu'en tant qu'organisme à but lucratif, les entreprises s'orientent vers la philanthropie, le mécénat devient alors un enjeu social (A). Au-delà, l'ambiguïté fondamentale du mécénat, consistant à mêler indistinctement l'intérêt public et celui de l'entreprise, aboutit à une structure d'accueil apparentée à un véritable instrument financier, permettant d'ancrer définitivement le mécénat dans la logique d'entreprise (B).

\footnotetext{
${ }^{36}$ C'est-à-dire soumis à la réalisation de volontés stipulées par le ou les fondateurs. Par exemple, lorsque le Duc D'aumale a légué le domaine de Chantilly à l'Institut de France, il l'a encadré par des dispositions testamentaires strictes, dont l'impossibilité de prêter les collections à l'extérieur ou encore d'en modifier les présentations.
} 


\section{A. L'instrument social des entreprises mécènes}

La fondation d'entreprise. Véhicule hybride ${ }^{37}$, en marge des fondations RUP trop lourdes et des fondations sous égides sclérosées dans des habits trop étroits, la fondation d'entreprise est née de la loi $\mathrm{n}^{\circ}$ 90-559 du 4 juillet 1990, dite "Loi Lang ${ }^{38}$. Mieux adaptée aux entreprises de petite ou de taille moyenne, elle est réservée aux sociétés civiles ou commerciales, aux établissements publics à caractère industriel et commercial, aux coopératives, ainsi qu'aux institutions de prévoyance ou aux mutuelles.

Si ces structures répondent aux mêmes exigences d'intérêt général et de but non lucratif que les fondations RUP, elles renouent avec la capacité juridique, ce qui accroît leur visibilité vis-à-vis des tiers et des bénéficiaires. Mais cette parenté se limite à ces caractères fondamentaux. Pour le reste, la fondation d'entreprise obéit à un régime singulier.

Elle présente en effet de sérieux atouts. Plusieurs assouplissements sont prévus pour faciliter leur création. Plus souple, sa dotation minimale est d'un cinquième des dépenses envisagées pour l'ensemble du programme sur cinq ans (minimum) et ne doit pas être inférieure à $152440 €$. Depuis 2002, il n'est même plus nécessaire qu'elles disposent d'une dotation initiale. Ce programme quinquennal s'aligne d'ailleurs sur la durée limitée de cette fondation, l'obligation de pérennité ayant été supprimée. Simplifiée, sa création résulte d'un simple arrêté préfectoral, et l'État est absent de son fonctionnement, écarté du conseil d'administration de la fondation.

En revanche, elle présente de nombreux inconvénients. Ainsi, contrairement aux fondations d'utilité publique, la fondation d'entreprise «ne peut acquérir ou posséder d'autres immeubles que ceux nécessaires au but qu'elle se propose $»^{39}$, ce qui la rapproche des associations ${ }^{40}$. De plus, toutes les valeurs

\footnotetext{
${ }^{37}$ R. Besnard Goudet, J.-Cl. civ. Annexe, V V Fasc. 20 : Fondations - Fondation d'entreprise, spéc. ${ }^{\circ} 1$.

${ }^{38}$ E. Alfanderi et M. Jeantin, « Fondation d'entreprise, loi du 4 juillet $1990 »$ : RTD com., 1990, p. 608; Y. Streiff, « La loi du 4 juillet 1990 créant les fondations d'entreprise et modifiant les dispositions sur le mécénat», Bull. Joly 1990, p.835 ; Ch. Debbasch, «Le nouveau statut des fondations : Fondations d'entreprise et fondations classiques », D. 1990, p. 269; O. Binder, "Aspects juridiques et fiscaux du mécénat d'entreprise » : Gaz Pal. 1987, 2, 744.

${ }^{39}$ L. $\mathrm{n}^{\circ}$ 87-571, 23 juill. 1987, art. 19-3, alinéa 1er (Créé par Loi n 90-559 du 4 juillet 1990).

${ }^{40}$ L. 1er juill. 1901 (art. 6 et 11).
} 
mobilières détenues par la fondation d'entreprise doivent respecter un certain formalisme ${ }^{41}$. En outre, lorsqu'elle «détient des actions des sociétés fondatrices ou de sociétés contrôlées par elles, la fondation ne peut exercer les droits de vote attachés à ces actions ${ }^{42}$, ceci afin d'éviter qu'elle n'use d'un pouvoir dépourvu de lien avec son objet d'intérêt général ${ }^{43}$. Enfin, la fondation d'entreprise se distingue de ses modèles par une capacité de collecte réduite dès lors qu'elle ne peut pas faire appel à la générosité publique, ni recevoir des legs et dons, manuels ou notariés, à peine de retrait de l'autorisation préfectorale ${ }^{44}$.

Or, fort opportunément, cet élément contrariant est, au fil des réformes, devenu un facteur d'identification des fondations d'entreprises. Lorsque la loi $\mathrm{n}^{\circ}$ 2003-709 du $1^{\text {er }}$ août 2003 relative au mécénat, aux associations et aux fondations, dite "loi Aillagon», a répondu au vœu du Président Chirac d'encourager et d'impliquer dans notre vie culturelle tous les acteurs de la société civile en portant l'essentiel de l'effort sur le régime fiscal ${ }^{45}$, elle n'a pas oublié d'améliorer cette structure sur le plan du financement. Elle a ainsi accru leur capacité juridique pour les autoriser à recevoir des dons non seulement de la part des salariés de l'entreprise fondatrice, mais encore de ceux accomplis par les salariés des entreprises du groupe auquel l'entreprise fondatrice appartient ${ }^{46}$, en plus d'imposer leur présence à sa gouvernance ${ }^{47}$. Cette loi favorise ainsi l'émergence des fondations d'entreprises en développant leur image d'entreprises citoyennes. Dans cette droit ligne, la loi n ${ }^{\circ} 2014-856$ du 31 juillet 2014 relative à l'économie sociale et solidaire a plus largement autorisé les dons de la part des mandataires sociaux, des sociétaires ainsi que des adhérents ou actionnaires de l'entreprise ou des entreprises du groupe.

\footnotetext{
${ }^{41}$ ils « doivent être placées en titres nominatifs, en titres pour lesquels est établi le bordereau de références nominatives prévu à l'article 55 de la loi n 87-416 du 17 juin 1987 sur l'épargne ou en valeurs admises par la Banque de France en garanties d'avances ». Ibidem.

${ }^{42}$ Ibid. Comp. : C.com., art. L. 233-31.

${ }^{43}$ Rapp. E. Dailly, 26 avr. 1990 : JO Sénat CR, 27 avr. 1990, p. 596.

${ }^{44}$ L. $n^{\circ} 87-571,23$ juill. 1987, art. 19-8.

45 Puisqu'il s'agissait de permettre notamment aux entreprises et aux particuliers de déduire respectivement $60 \%$ et $66 \%$ de leurs dépenses de mécénat de leurs impôts, sous certaines conditions.

${ }^{46} \mathrm{Au}$ sens de l'article $223 \mathrm{~A}$ du CGI.

${ }^{47}$ Le conseil d'administration doit comporter au moins deux salariés de l'entreprise fondatrice de manière à «asseoir leur légitimité à l'intérieur de l'entreprise » et recueillir une véritable adhésion en son sein.
} 
Resserrée sur la générosité de ses parties prenantes, la fondation d'entreprise devient le tremplin à la quête de «citoyenneté » poursuivie par les entreprises animées par une démarche RSE. Rien d'étonnant, dès lors, à ce qu'elles aient intégré le mécénat comme stratégie majeure de leur développement afin de remplir leur devoir sociétal en « labellisant» leur action culturelle comme une action sociale. Qu'il s'agisse de consolider le lien entre l'entreprise, les citoyens et les salariés, de mobiliser ces derniers autour d'un projet commun, de les fidéliser, de renforcer les synergies dans l'entreprise, de développer une identité d'entreprise au sein d'un groupe de dimension internationale, sinon de participer à la moralisation de leurs activités, c'est dire si les mérites de cette structure sont nombreux !

Principalement incarné par les secteurs de la banque, de la finance et de l'assurance, ce type de fondation se montre davantage soucieux de l'environnement au sein duquel les entreprises donatrices opèrent. Elles participent ainsi au renouvellement d'un mécénat de proximité ${ }^{48}$, même si certaines connaissent d'un rayonnement national ${ }^{49}$.

\section{B. Les instruments financiers des entreprises mécènes}

Les fonds de dotation. Dernière structure créée par la loi n 2008-776 du 4 août 2008 de modernisation de l'économie ${ }^{50}$ pour compléter la gamme des outils dédiés au mécénat, le fonds de dotation fait une intrusion remarquée en droit français $^{51}$. Sa création peut être à l'initiative d'une société commerciale ou de l'associé de celle-ci, puisque l'article 140, I de la loi susvisée prévoit que toute personne physique et morale peut créer un fonds de dotation.

En tant que personne morale de droit privés2 à but non lucratif, le fonds de dotation reçoit et gère, en les capitalisant, des biens et droits de toute nature, qui

\footnotetext{
${ }^{48}$ C'est ainsi qu'à Toulouse, la fondation Espace Écureuil est très active. De même, les Chorégies d'Orange sont aidées par France Télécom.

${ }^{49}$ La Fondation Louis Vuitton fait actuellement le plein avec l'exposition de la Fondation Chtchoukine.

${ }^{50}$ L. $n^{\circ}$ 2008-776, 4 août 2008, de modernisation de l'économie, art. 140. I : JO, 5 août 2008, p. 12471.

${ }^{51}$ F. Perrotin, « Fonds de dotation : des créations toujours plus nombreuses », LPA, 10 août 2011, p. 3.

52 Sur les modalités de constitution, voir: Circulaire du 19 mai 2009 relative à l'organisation, au fonctionnement et au contrôle des fonds de dotation : NOR:ECEM0908677c, JORF n 0140 du 19 juin 2009, p. 9992, texte $n^{\circ} 23$.
} 
lui sont apportés à titre gratuit et irrévocable, et utilise les revenus de capitalisation soit directement, au service d'une œuvre ou d'une mission d'intérêt général, soit, indirectement, en les distribuant à un autre organisme à but non lucratif. Inspiré des «endowment funds» anglo-saxons, il se présente ainsi comme un outil de capitalisation financière au service de l'intérêt général.

Ce faisant, il répond à un double défi : concilier le prestige des fondations, tel que révélé par leur capacité de collecte et les larges perspectives qu'elles offrent, avec la souplesse ainsi que la popularité des associations : création par déclaration préfectorale ${ }^{53}$, absence de dotation initiale minimale, libre gouvernance ${ }^{54}$, en sont les éléments clefs.

Rien d'étonnant, dès lors, à ce que les associations et les fondations se soient inquiétées de la «rivalité ${ }^{55}$ que semblait leur faire ce nouvel outil dédié au mécénat et à la philanthropie. Pourtant, si les fonds de dotation s'inspirent de ces modèles, ils s'en éloignent par de nombreux d'aspects.

D'ailleurs, par crainte que ne se multiplient les «coquilles vides", le caractère facultatif de la dotation initiale a ensuite été corrigé par la loi $n^{\circ} 2014$ $856 \mathrm{du} 31$ juillet $2014^{56}$. Reste que cette obligation peut aisément être contournée dès lors que la dotation d'un fonds peut être consomptible si les statuts le stipulent ${ }^{57}$. Dans cette hypothèse, la dotation initiale pourra être consommée en totalité durant la vie du fonds pour la réalisation de la mission du fonds. En ce cas, ils deviennent inéligibles à la réduction ISF prévue par la loi $\mathrm{n}^{\circ}$ 2007-1233 du 21 août 2007, dite Loi TEPA ${ }^{58}$.

\footnotetext{
${ }^{53}$ Décret n ${ }^{\circ} 2009-158$ du 11 février 2009.

${ }^{54}$ Il est administré par un conseil d'administration dont la composition est libre, la loi se bornant à préciser que le conseil d'administration doit comporter au moins trois membres.

${ }^{55}$ C. Bergeal, « N'ayez pas peur des fonds de dotation », Jurisassociations, 15 mars 2009, $\mathrm{n}^{\circ} 395$.

${ }^{56}$ Cette loi a modifié l'article 140 de la loi LME instituant les fonds de dotation (D. $\mathrm{n}^{\circ} 2009-158$, 11 févr. 2009, relatif aux fonds de dotation). Précisant que cette dotation initiale sera « au moins égale à un montant fixé par voie réglementaire, qui ne peut excéder $30000 € »$, elle a laissé au décret $n^{\circ} 2015$ 49 du 22 janvier 2015 le soin d'en fixer le montant à 15000 euros. Des sanctions sont prévues en cas de manquement à cette obligation de dotation minimale (suspension du fonds de dotation, voire sa dissolution judiciaire). Comp. : F. Perrotin, « Encadrement des fonds de dotation », LPA 26 janv. 2015, $\mathrm{n}^{\circ} 18$, p. 4.

${ }^{57}$ Y. Vincent, « Fonds de dotation : les clauses statutaires importantes », Dr. sociétés 2014, form. 1.

${ }^{58}$ CGI, art. 885-0 V bis.
} 
Surtout, le prestige des fondations sort renforcé étant donné que, par principe, il leur est interdit de recevoir des fonds publics. De telle sorte que, sauf cas exceptionnel ${ }^{59}$, le fonds de dotation naît et vit de l'initiative privée.

Mais ces réserves n'empêchent pas ces fonds de remporter un réel succès ${ }^{60}$ ! Ils sont d'ailleurs l'objet d'une attention législative permanente.

Ces fonds ne procèdent pas des mêmes enjeux et n'obéissent pas à la même philosophie. Le fonds de dotation n'est pas une institution sociale. Il ne traduit pas une volonté de vivre en commun, de définir un lieu de sociabilité et de partage, comme peuvent l'être une association, une fondation et, a fortiori, une fondation d'entreprise. Il reste un instrument financier doté de la personnalité juridique qui permet à son ou à ses fondateurs de collecter des fonds, renouant ainsi avec la fonction première de l'entreprise, si ce n'est quant à l'utilisation qui en est faite. Pour autant ses mérites évoluent et rejoignent parfois les préoccupations précédentes. De plus en plus de fonds de dotation permettent aux entreprises de prolonger leur responsabilité sociale dans le cadre du mécénat ou interviennent dans une optique de transmission en jouant le rôle d'une holding, lorsque le chef d'entreprise souhaite réaliser une opération philanthropique à cette occasion ${ }^{61}$.

Les fondations actionnaires d'entreprises. Après 8 ans d'immobilisme, fin septembre 2016 se tenait à Bercy la première conférence européenne sur les «fondations actionnaires d'entreprises $»^{62}$. L'expression désigne des fondations propriétaires d'entreprises. Il n'est point d'oxymore. Au contraire, ce modèle réconcilie les prétendus inconciliables : philanthropie et capitalisme.

\footnotetext{
${ }^{59}$ Quant à se prévaloir d'une exception, le formalisme à respecter (double arrêté ministériel) suffit à dissuader. Seul le fonds de dotation du Louvre en a bénéficié.

${ }^{60}$ On en comptait 852 en 2011, ils sont 2226 en 2016 selon les statistiques publiées par l'Observatoire de la Fondation de France au 31 décembre 2016. Comp. : E. Rocher, «Les fonds de dotation : un véritable paradis fiscal pour la philanthropie », La Tribune, 3 sept. 2014. Y. Vincent, "Le fonds de dotation, où comment concilier philanthropie et optimisation fiscale: un outil à (re)découvrir », Dr. sociétés janv. 2014, p. 5.

${ }^{61}$ R. Vabres, «Le fonds de dotation peut-il être un outil de transmission des entreprises ? », Defrénois, 15 sept. 2014, n 17, p. 911.

${ }^{62}$ C. Bach, «Les fondations sont-elles les actionnaires de demain? », Option Droit et Affaires, sept. 2016.
} 
Selon ce modèle, ce n'est plus l'entreprise qui alloue une part, souvent infime, de ses résultats à une fondation périphérique, mais la fondation reconnue d'utilité publique, elle-même, qui possède l'entreprise industrielle ou commerciale, en détenant tout ou partie des actions ou parts sociales et la majorité des droits de vote et/ou la minorité de blocage, de telle sorte que la fondation oriente ses investissements, décide de sa stratégie, et finance, grâce aux dividendes qu'elle perçoit, des causes d'intérêt général, lesquels, il est vrai, font souvent écho à l'objet social de l'entreprise ainsi détenue ${ }^{63}$.

Malgré les réticences d'un Conseil d'État soucieux de méconnaître le «principe de spécialité ${ }^{64}$ en «contaminant» la mission d'intérêt général confiée à la fondation, cette figure a finalement été actée dans la loi $\mathrm{n}^{\circ} 2005-882$ du 2 août 2005 en faveur des PME, dite « loi Dutreil » ${ }^{65}$. Son article 29 ouvre à une fondation reconnue d'utilité publique la possibilité de détenir des titres de société ou, inversement, pour une entreprise d'apporter des titres sociaux à une fondation.

Toutefois, ce levier philanthropique puissant peine à séduire en France ${ }^{66}$, là où en Europe, les exemples se multiplient ${ }^{67}$ ! Différents verrous freinent encore son expansion.

Pourtant ce modèle de transmission et de gouvernance des entreprises semble vertueux en ce qu'il permet notamment de protéger le capital de l'entreprise qu'elle détient, puisque celui-ci lui a été transmis de façon

\footnotetext{
${ }^{63}$ Par ex., la vie culture du canton de Genève est essentiellement financée par la Fondation Wilsdorf, propriétaire à $100 \%$ de Rolex.

${ }^{64}$ Pendant longtemps, le Conseil d'Etat a en effet considéré qu'une fondation RUP ne pouvait pas détenir des participations dans une entreprise, du moins de manière autre qu'à titre de simple placement financier accessoire. Selon lui, il ne serait pas dans l'objet de ce type d'organisation non lucrative de gérer une entreprise, qui pourrait en effet "contaminer" son intérêt général; et faire courir un risque à la fondation si, face à des difficultés économiques, il fallait soit recapitaliser la société filiale, soit la liquider.

${ }^{65}$ L. n ${ }^{\circ}$ 2005-882, 2 août 2005, en faveur des petites et moyennes entreprises (art. 29) modifiant L. $n^{\circ}$ 87-571, 23 juill. 1987, sur le développement du mécénat, (art. 18-3).

${ }^{66}$ Le pionnier en France fut la Fondation Pierre Fabre.

${ }^{67}$ Ce modèle est plébiscité par de nombreux états de l'Europe du Nord, principalement le Danemark, la Norvège, la Suède, l'Allemagne. Ainsi Carlsberg, Ikéa, Rolex, Sandoz, Bosch, Playmobil, Bertlesmann, Novo Nordisk... appartiennent en totalité ou en majorité à des fondations. Il en est de même de Tata en Inde.
} 
irrévocable et inaliénable. Par cette irrévocabilité, cette figure de la fondation actionnaire s'adresse donc en priorité à des entrepreneurs désintéressés, philanthropes et sans héritier direct. Par son inaliénabilité, elle assure l'indépendance et la pérennité de l'entreprise en la protégeant des OPA hostiles en la tenant à l'abri des spéculations. Surtout, ce modèle assure à la fondation des revenus réguliers et conséquents, lui permettant de « réconcilier les fruits du capital avec l'intérêt général ${ }^{68}$.

C'est aussi l'occasion de vivre autrement l'entreprise, puisque la valeur créée par celle-ci concourt de façon significative à une action philanthropique.



L'argent et le talent. Achever cette étude par l'examen de cette figure singulière permet surtout de rendre compte du déploiement d'une philanthropie plus entrepreneuriale et récuse définitivement la vision manichéenne avancée en propos introductifs consistant à douter de l'alliance de la philanthropie au capitalisme au service de la culture. Loin de s'exclure, l'un et l'autre s'alimentent réciproquement en créant des liens profonds entre des mondes qui tendaient à s'ignorer. L'idée d'une «entreprise mécène» est de la sorte définitivement actée. Ceci est d'autant plus précieux en période de crise économique, alors que le sens commun incline parfois à penser la culture comme un luxe et à voir dans son financement une dépense superfétatoire !

Sans rompre avec les préceptes anciens, l'essor d'une philanthropie moins étatique et, partant, plus entrepreneuriale, permet de renouveler les voies de son financement en multipliant, à l'image d'un tableau impressionniste, par retouches successives, les facteurs de rencontre du talent des uns et de l'argent des autres. C'est donc reconnaître qu'en moins de vingt ans, fort du potentiel qu'il représente, le mécénat d'entreprises est devenu l'un des outils fondamentaux du développement de l'économie culturelle en France.

Assurément, en est-il ainsi de ces entreprises avec un supplément d'art !

\footnotetext{
${ }^{68}$ V. Seghers, «Les fondations actionnaires d'entreprises : allier stabilité et performance durable », L'ISR Way, La gouvernance, p.12 et s.
} 